\title{
Heat Shock Protein 70 and 90 Genes in the Harmful Dinoflagellate Cochlodinium polykrikoides: Genomic Structures and Transcriptional Responses to Environmental Stresses
}

\author{
Ruoyu Guo, ${ }^{1}$ Seok Hyun Youn, ${ }^{2}$ and Jang-Seu Ki ${ }^{1}$ \\ ${ }^{1}$ Department of Life Science, Sangmyung University, Seoul 110-743, Republic of Korea \\ ${ }^{2}$ Fishery and Ocean Information Division, National Fisheries Research \& Development Institute, Busan 619-705, Republic of Korea \\ Correspondence should be addressed to Jang-Seu Ki; kijs@smu.ac.kr
}

Received 18 November 2014; Accepted 31 March 2015

Academic Editor: Elena Pasyukova

Copyright (C) 2015 Ruoyu Guo et al. This is an open access article distributed under the Creative Commons Attribution License, which permits unrestricted use, distribution, and reproduction in any medium, provided the original work is properly cited.

\begin{abstract}
The marine dinoflagellate Cochlodinium polykrikoides is responsible for harmful algal blooms in aquatic environments and has spread into the world's oceans. As a microeukaryote, it seems to have distinct genomic characteristics, like gene structure and regulation. In the present study, we characterized heat shock protein (HSP) 70/90 of C. polykrikoides and evaluated their transcriptional responses to environmental stresses. Both HSPs contained the conserved motif patterns, showing the highest homology with those of other dinoflagellates. Genomic analysis showed that the CPHSP70 had no intron but was encoded by tandem arrangement manner with separation of intergenic spacers. However, CPHSP90 had one intron in the coding genomic regions, and no intergenic region was found. Phylogenetic analyses of separate HSPs showed that CpHSP70 was closely related with the dinoflagellate Crypthecodinium cohnii and CpHSP90 with other Gymnodiniales in dinoflagellates. Gene expression analyses showed that both HSP genes were upregulated by the treatments of separate algicides $\mathrm{CuSO}_{4}$ and $\mathrm{NaOCl}$; however, they displayed downregulation pattern with PCB treatment. The transcription of CPHSP90 and CPHSP70 showed similar expression patterns under the same toxicant treatment, suggesting that both genes might have cooperative functions for the toxicant induced gene regulation in the dinoflagellate.
\end{abstract}

\section{Introduction}

Dinoflagellate algae are a eukaryotic protist and are the most important primary producer in aquatic environments. Some species (e.g., Alexandrium tamarense, Amphidinium carterae, Akashiwo sanguinea, Cochlodinium polykrikoides, Gymnodinium, and Karlodinium micrum) are responsible for harmful algal blooms (HABs), affecting fisheries and creating associated economic loss in aquaculture industries [1]. The ichthyotoxic Cochlodinium polykrikoides is one of the most common dinoflagellates that cause HABs, and it has expanded oceanic regions worldwide $[2,3]$. Thus, its bloom can cause severe environmental impacts and huge economic losses, due to lots of fish mortalities in aquaculture [4-6]. In the last three decades, the causative organism has been extensively studied in terms of environmental survey, bloomforming mechanisms, and/or mitigation measures [7-11]; nevertheless, some issues, like toxic mechanisms and cellular gene response, still remained unclear. Molecular study is very useful and crucial for understanding regulation mechanism and molecular characteristics of the causative organism. However, molecular studies of C. polykrikoides especially in terms of toxicogenomics and gene regulation are limited so far.

Heat shock proteins (HSPs) are remarkably evolutionary conserved molecular chaperones and are present in all the prokaryotic and eukaryotic organisms. They are distributed into small HSP, HSP60, HSP70, HSP90, and HSP100, depending on their molecular weight and sequence similarity [12]. HSPs have multiple roles, including membrane translocation, protein degradation, protein folding, and repair misfolded proteins, in regulation of protein homeostasis in normal and stressed cells for regulating protein homeostasis in normal and stressed cells $[12,13]$. Hence, HSPs are one of the major 
genes that can be induced and respond to various stressors, and, of them, HSP70 may be firstly induced under stress conditions rather than other HSPs [12]. Moreover, HSP70 and HSP90 are the most conserved and abundant HSPs and are widely involved in the environmental stressors, such as thermal shock, heavy metal, oxidative damage, hypoxia, and xenobiotic chemicals [12, 14-16]. For this reason, either HSP90 or HSP70 is considered as biomarker for environmental monitoring $[17,18]$. Furthermore, HSP90 and HSP70 may interact with each other, as well as cooperate with other HSPs or chaperone to regulate multisignal transduction pathway [19-21].

In cases of the dinoflagellates, several of HSP90 and HSP70 genes have been identified, but majority of researches have focused on phylogenetic relationships or spliced leader sequence analyses in dinoflagellates [22-25]. Only few studies have investigated the responses of HSP90 and/or HSP70 under environmental stress conditions [26-28]. For example, we reported HSP70/90 from the dinoflagellates Prorocentrum minimum, suggesting that both genes play diverse roles in physiological responses of the dinoflagellate $[27,28]$. So, it is necessary to discover more molecular information for understanding gene regulation mechanisms in adaptive, survival strategies of dinoflagellates, as well as gene and genomic structures. In the present study, we determined full length sequences of HSP90 and HSP70 of the dinoflagellate C. polykrikoides and characterized their gene and genomic features. These included analysis of genomic DNA, deduced protein sequences, phylogenetic relationships, and their gene regulation under metal and nonmetal stress conditions as well.

\section{Materials and Methods}

2.1. Cell Culture. C. polykrikoides was obtained from the National Fisheries Research and Development Institute (NFRDI), Korea. The C. polykrikoides cells were cultured in $\mathrm{f} / 2$ medium at $20^{\circ} \mathrm{C}$ in 12:12 h light-dark cycle, with a photon flux density of about $65 \mu \mathrm{mol}$ photons $\mathrm{m}^{-2} \mathrm{~s}^{-1}$.

\subsection{RNA Extraction, cDNA Synthesis, and DNA Extraction.} C. polykrikoides cultures were harvested by centrifugation at $1,000 \mathrm{~g}$ for $10 \mathrm{~min}$, frozen immediately in liquid nitrogen and stored at $-80^{\circ} \mathrm{C}$ until RNA extraction. Preserved cells were physically broken by freeze-thawing in liquid nitrogen and further homogenized by a Mini-Beadbeater (BioSpec Products Inc., Bartlesville, OK) with zirconium beads (diameter $0.1 \mathrm{~mm}$ ). Total RNA was isolated using the TRIzol (Invitrogen, Carlsbad, CA) and purified by Mini Spin Columns of RNeasy Mini Kit (Qiagen, Valencia, CA). For the first strand cDNA, 2 different cDNA synthesis kits were employed: one was SuperScript III First-Strand Synthesis System (Invitrogen, Carlsbad, CA) for the gene cloning of CpHSP70 and CpHSP90; the other was a Maxime RT PreMix Kit with random primers (iNtRON, Seongnam, Republic of Korea) for gene expression study. Then, the 1st strand cDNA templates were diluted $1: 10$ with nuclease-free water for use in subsequent analyses. Total genomic DNA was extracted from C. polykrikoides following cetyltrimethylammonium bromide (CTAB) [29].

2.3. Gene Sequences Determination. Full length of CpHSP70 and CPHSP90 sequences was determined by rapid amplification of cDNA ends (RACE). Partial gene sequences of CpHSP70 and CPHSP90 were taken from C. polykrikoides EST database (GenBank accession number SRR1917383) determined by 454 pyrosequencing (GS-FLX Titanium; 454 Life Sciences, Roche, Branford, CT). CpHSP70 and CpHSP90 EST sequences were used for primer design for full length amplification (Table 1 ). The $3^{\prime}$ - and $5^{\prime}$-untranslated regions (UTR) of these genes were determined by using the $3^{\prime}$ and $5^{\prime}$-RACE, respectively. For the RACE, nest PCRs were employed, and the primers used in each PCR were listed in Table 1. Reaction conditions for the primary and secondary PCRs were as follows: predenaturation at $96^{\circ} \mathrm{C}$ for $10 \mathrm{~min} ; 35$ cycles of $95^{\circ} \mathrm{C}$ for $30 \mathrm{~s}, 52^{\circ} \mathrm{C} / 54^{\circ} \mathrm{C}$ for $30 \mathrm{~s}, 72^{\circ} \mathrm{C}$ for $100 \mathrm{~s}$, and extension at $72^{\circ} \mathrm{C}$ for $10 \mathrm{~min}$, respectively. Positive core PCR products were purified, cloned into pMD20-T vector (Takara, Shiga, Japan), transformed into E. coli competent cells, and subjected to sequencing. The full length of the CpHSP70 and $C$ PHSP90 was validated by PCR with specific primers (Table 1). The primers used in the CpHSP70 and CpHSP90 genomic sequence determination were designed according to cDNA sequence (Table 1).

2.4. CpHSP70 and CpHSP90 Characterization and Phylogenetic Analysis. Protein motifs and conserved domains of CPHSP70 and CPHSP90 protein were analyzed with the online servers and public database, including the PROSITE (http://prosite.expasy.org/), Compute pI/Mw tool (http://web.expasy.org/compute_pi/), and NCBI Conserved Domain Database (http://www.ncbi.nlm.nih.gov/Structure/ cdd/wrpsb.cgi).

Phylogenetic analysis was performed in MEGA5 [30], using the neighbor-joining method [31]. Bootstrap consensus tree inferred from 1,000 replicates was taken to represent the evolutionary history of the taxa analyzed [32]. The tree is drawn to scale, with branch lengths in the same units as those of the evolutionary distances used to infer the phylogenetic tree. The evolutionary distances were computed using the JTT matrix-based method [33] and were in the units of the number of amino acid substitutions per site. In the sequences analysis, all positions containing gaps and missing data were eliminated. It involved 28 amino acid sequences and had a total of 489 positions in the final HSP90s dataset. In the case of HSP70s, it involved 23 amino acid sequences. There were a total of 586 positions in the final HSP70s dataset.

\subsection{Toxicant Treatments, Gene Expression, and Statistical} Analysis. Exponential phase cells were used for toxicant treatments. Typical toxicants $\mathrm{CuSO}_{4}$ (Cat. number C1297, Sigma, MO), $\mathrm{NaOCl}$ (Cat. number 425044, Sigma, MO), and Aroclor 1016 (48701, Sigma, a type of PCBs) were employed in the present study. To test the doses effect of toxicants on CpHSP70 and CPHSP90 transcriptional expression, a series of concentrations of each toxicant were added in the $C$. polykrikoides cultures (with final concentration of $\mathrm{CuSO}_{4}: 1$, 
TABLE 1: The primers used in the present study.

\begin{tabular}{|c|c|c|c|}
\hline Gene & Primer & Remark & Nucleotide sequence $\left(5^{\prime} \rightarrow 3^{\prime}\right)$ \\
\hline CpHSP90 & CpH90F1 & RT-PCR & GTTCGACACCTCACTGCTCACC \\
\hline CpHSP90 & CpH90R1 & RT-PCR & AGGCCGAGCTTGATCATGC \\
\hline CpHSP70 & $\mathrm{CpH70F1}$ & RT-PCR & GGGCAGACCTTCACGACGTATG \\
\hline CpHSP70 & CpH70R1 & RT-PCR & AATGCCGTCCAAGTGGAACTTC \\
\hline Cp18S & Cp18S-F & RT-PCR & GGAGTATGGTCGCAAGGCTGAAAC \\
\hline Cp18S & Cp18S-R & RT-PCR & CCTCGTGTTGAGTCAAATTAAGCC \\
\hline СpHSP90 & CpHSP90-3F2 & $3^{\prime}-\mathrm{RACE}$ & TGCTCACCTCCGGCTTCAAC \\
\hline CpHSP90 & CpHSP90-3F3 & $3^{\prime}$-RACE & CCACCGCATGATCAAGCTC \\
\hline CpHSP90 & CpHSP90-5R2 & $5^{\prime}-\mathrm{RACE}$ & ATGCGGCTGCTGATGATG \\
\hline CpHSP90 & CpHSP90-5R3 & $5^{\prime}-\mathrm{RACE}$ & АССТTCTCCACCTTGTCGC \\
\hline CpHSP90 & CpHSP90-SR1 & Full length & GTGTCCAAGTCTTGTGATCGAC \\
\hline CpHSP90 & CpHSP90-SR2 & Full length & AGCACTAGCGCCGGTCATGT \\
\hline CpHSP90 & 5-SL & $5^{\prime}$-RACE/full length & CGTAGCCATTTTGGCTCAAG \\
\hline CpHSP70 & CpHSP70-3F1 & $3^{\prime}-\mathrm{RACE}$ & CGCTGCAGGAGGAGAAGTTG \\
\hline CpHSP70 & CpHSP70-3F2 & $3^{\prime}-\mathrm{RACE}$ & TGGAGGGCGTTGTCAATC \\
\hline CpHSP70 & CpHSP70-SF1 & Full length & TTTCTCGGGGGTTTTGCTTCG \\
\hline CpHSP70 & CpHSP70-SF2 & Full length & CATGGCGAAAAAGACGGCTGTT \\
\hline CpHSP70 & CpHSP70-SR1 & Full length & TGGGACAGTCTGGGGTTGCT \\
\hline \multirow[t]{3}{*}{ CpHSP70 } & CpHSP70-SR2 & Full length & ACACTCGGCGAATCCTCAG \\
\hline & B26 & $3^{\prime}-\mathrm{RACE}$ & GACTCTAGACGACATCGA(T) $)_{18}$ \\
\hline & B25 & $3^{\prime}-\mathrm{RACE}$ & GACTCTAGACGACATCGA \\
\hline CpHSP70 & CpHSP70-DF1 & Genomic DNA & TGTCAGTCATCCCCTATTTGTC \\
\hline CpHSP70 & CpHSP70-DR1 & Genomic DNA & GACAGTCTGGGGTTGCTGAATT \\
\hline CpHSP90 & CpHSP90-DF1 & Genomic DNA & TGGGAAACCATTTTTGAGCATTG \\
\hline CpHSP90 & CpHSP90-DR1 & Genomic DNA & TGCAGGTGTGAACCACTCAGC \\
\hline CpHSP70 & CpHSP70-IF1 & Intergenic DNA & GAGGGCGTTGTCAATCCCATG \\
\hline CpHSP70 & CpHSP70-IR1 & Intergenic DNA & AATGCCGTCCAAGTGGAACTTC \\
\hline
\end{tabular}

5, and $8 \mathrm{mg} \mathrm{L}^{-1} ; \mathrm{NaClO}_{3}: 0.02,0.1,0.3$, and $0.5 \mathrm{mg} \mathrm{L}^{-1}$; PCB: $0.05,0.1,0.2$, and $\left.0.5 \mathrm{mg} \mathrm{L}^{-1}\right)$. The treated and untreated cultures were harvested for gene expression analysis at indicated time points. RNA extraction and cDNA were prepared with the same manner described previously. Gene expression and statistical analysis were followed by Guo et al. [27].

\section{Results and Discussion}

3.1. CpHSP90 Characteristics and Phylogeny. CpHSP90 (GenBank number KP010829) was 2,316 bp in length, coding 709 amino acids (aa) with theoretical isoelectric point (pI) 4.9 and molecular weight $(\mathrm{Mw}) 81.7 \mathrm{kDa}$. Its deduced protein shared the highest sequence similarity (681 identities in 709 amino acids) with those of the dinoflagellate Prorocentrum minimum (HSP90, GenBank number AFD34191), followed by Karlodinium veneficum with 665 identities in 709 aa (HSP90; ABI14419). Generally, the HSP90 contains five conserved motifs defined as HSP90 signature motif [34]; these five signature motifs NKEIFLRELISNASDALDKIRY, LGTIAKSGT, IGQFGVGFYSAYLV, IKLYVRRVFI, and VVDSEDLPLNISRE were identified by comparison with other HSP90s (Figure 1(a)). Furthermore, the conserved MEEVD was identified in the C-terminus of deduced CpHSP90, which indicated that CpHSP90 protein belongs to the cytosolic HSP90 family [35].

Phylogenetic analysis was performed using CpHSP90 protein and other dinoflagellate HSP90 proteins (Figure 1(b)). A resultant tree showed C. polykrikoides with Gymnodinium fuscum and Lepidodinium chlorophorum was clustered into one clade, of which taxon position belonged to the order Gymnodiniales. Furthermore, other dinoflagellate orders like Perdiniales were spited into two clades as well. These results suggested that the dinoflagellate HSP90s explosive scattered in morphology and diversity [24].

3.2. CpHSP70 Characteristics and Phylogeny. In addition, full ORF of CPHSP70 (GenBank number KP010828) was 1,944 bp in length, coding 648 aa with theoretical pI 5.12 and Mw $70.8 \mathrm{kDa}$. CpHSP70 aa showed $94 \%$ maximum identity with those of the dinoflagellates Crypthecodinium cohnii (GenBank number AAM02973) and Prorocentrum minimum (ABI14407), followed by $88 \%$ identity with Perkinsus marinus (XP_002780413). We identified three HSP70 motifs, IDLGTTYS, IYDMGGGTFDVSLL, and VVLVGGSTRIPKVQS, in this protein (Figure 2(a)). In addition, the EEVD motif was identified in the CpHSP70 protein C-terminus, which 
-87 CGTAGCCATTTT

GGCTCAAGATTCGGCGTCGCTGGGAAACCATTTTTGAGCATTGTTTGCCTGAGGGGTATCCGACCATTTTAGGAA ATGTCGGAGACATTCGCA TTCAATGCTGACATCCAGCAGCTGATGAGCTTGATCATCAACACCTTCTACAGCAAC $\begin{array}{llllllllllllllllllllllllll}M & \mathrm{~S} & \mathrm{E} & \mathrm{T} & \mathrm{F} & \mathrm{A} & \mathrm{F} & \mathrm{N} & \mathrm{A} & \mathrm{D} & \mathrm{I} & \mathrm{Q} & \mathrm{Q} & \mathrm{L} & \mathrm{M} & \mathrm{S} & \mathrm{L} & \mathrm{I} & \mathrm{I} & \mathrm{N} & \mathrm{T} & \mathrm{F} & \mathrm{Y} & \mathrm{S} & \mathrm{N}\end{array}$ AAAGAGA TCTTCCTCCGAGAGTTGATCAGCAACGCATCCGATGCATTGGACAAGA TCAGGTACGAGTCCATCACC $\begin{array}{lllllllllllllllllllllllll}\mathrm{K} & \mathrm{E} & \mathrm{I} & \mathrm{F} & \mathrm{L} & \mathrm{R} & \mathrm{E} & \mathrm{L} & \mathrm{I} & \mathrm{S} & \mathrm{N} & \mathrm{A} & \mathrm{S} & \mathrm{D} & \mathrm{A} & \mathrm{L} & \mathrm{D} & \mathrm{K} & \mathrm{I} & \mathrm{R} & \mathrm{Y} & \mathrm{E} & \mathrm{S} & \mathrm{I} & \mathrm{T}\end{array}$ GATCCGGACAAGATCGAGGCGCAGCCGAACTTTTTCATCAAAATTGTTCCGGACAAGACGAACTCGACCTTGACC $\begin{array}{llllllllllllllllllllllllll}\mathrm{D} & \mathrm{P} & \mathrm{D} & \mathrm{K} & \mathrm{I} & \mathrm{E} & \mathrm{A} & \mathrm{Q} & \mathrm{P} & \mathrm{N} & \mathrm{F} & \mathrm{F} & \mathrm{I} & \mathrm{K} & \mathrm{I} & \mathrm{V} & \mathrm{P} & \mathrm{D} & \mathrm{K} & \mathrm{T} & \mathrm{N} & \mathrm{S} & \mathrm{T} & \mathrm{L} & \mathrm{T}\end{array}$ ATCGAGGATTCTGGCATTGACATGACGAAGAACGAGTTGGTGAACAATCTGGGCACCATCGCGAAGTCTGGCACC $\begin{array}{lllllllllllllllllllllllllll}I & E & D & S & G & I & D & M & T & K & N & E & L & V & N & N & L & G & T & I & A & K & S & G & T\end{array}$ AAGGCATTCATGGAGGCCATGGCAGCCGGTGGCGACATCTCGATGATCGGGCAGTTCGGCGTGGGATTCTATTCC $\begin{array}{lllllllllllllllllllllllll}K & A & F & M & E & A & M & A & A & G & G & D & I & S & M & I & G & Q & F & G & V & G & F & Y & S\end{array}$ GCATACCTCGTGTCCGACAAGGTGCGCGT GATCAGCAAGCACAACGATGACGAGCAGTACATCTGGGAGTCGGCA $\begin{array}{llllllllllllllllllllllllll}A & Y & \text { L } & \text { V } & \text { S } & \text { D } & \text { K } & \text { V } & R & \text { V } & \text { I } & \text { S } & \text { K } & \text { H } & \text { N } & \text { D } & \text { D } & \text { E } & \text { Q } & \text { Y } & \text { I } & \text { W } & \text { E } & \text { S } & \text { A }\end{array}$ GCAGGAGGCTCCTTCACGGTGCAGAAGGACACCGAGCAGGTGCACGGTGAGGTGAAGCGCGGCACCAAGGTCATC $\begin{array}{llllllllllllllllllllllllll}A & G & G & S & F & T & V & Q & K & D & T & E & Q & V & H & G & E & V & K & R & G & T & K & V & I\end{array}$ TGCTACTTGAAGGAGGATCAGTCCGAGTTCCTTGAGGAGCGACGTTTGAAGGACTTGGTGAAGAAACACTCCGAG $\begin{array}{lllllllllllllllllllllllllllllllllllll}C & Y & L & K & E & D & Q & S & E & F & L & E & E & R & R & L & K & D & L & V & K & K & H & S & E\end{array}$ TTCA TCGGCTTCCCCA TCGAGCTGTACGTGGAGAAGTCGAAGGAGAAGGAGGTGACAGACAGCGAGGATGAGGAG

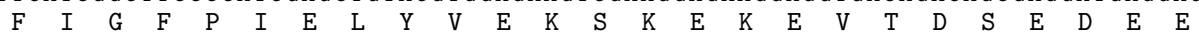
GAGGAGAAGAAGGAGGAGGATAAGGAGGGCGATGAGCCGAAGATCGAGGAGGTGGACGAGGAGAAGGAGAAGGAG $\begin{array}{llllllllllllllllllllllllllllllllllll}E & E & K & K & E & E & D & K & E & G & D & E & P & K & I & E & E & V & D & E & E & K & E & K & E\end{array}$ GAGAAGAAAAAGAGACCAAGAAGGTGAAGGAGGTGTCGCACGAGTGGGAGCAGCTCAACAAGAACAAGCCTCTG $\begin{array}{llllllllllllllllllllllllllll}E & K & K & K & K & T & K & K & V & K & E & V & S & H & E & W & E & Q & L & N & K & N & K & P & L\end{array}$ TGGATGCGCAAGTCGGAAGACGTGACCAACGAGGAGTATGCGTCGTTCTACAAGTCTCTGTCCAACGACTGGGAG $\begin{array}{lllllllllllllllllllllllllllll}W & M & R & K & S & E & D & V & T & N & E & E & Y & A & S & F & Y & K & S & L & S & N & D & W & E\end{array}$ GACCACCTGTCCGTCAAGCACTTCAGCGTTGAGGGCCAGCTTGAATTCCGTGCGTTGCTCTTCGTGCCTAGGCGT $\begin{array}{lllllllllllllllllllllllllll}\mathrm{D} & \mathrm{H} & \mathrm{L} & \mathrm{S} & \mathrm{V} & \mathrm{K} & \mathrm{H} & \mathrm{F} & \mathrm{S} & \mathrm{V} & \mathrm{E} & \mathrm{G} & \mathrm{Q} & \mathrm{L} & \mathrm{E} & \mathrm{F} & \mathrm{R} & \mathrm{A} & \mathrm{L} & \mathrm{L} & \mathrm{F} & \mathrm{V} & \mathrm{P} & \mathrm{R} & \mathrm{R}\end{array}$ GCTCCTTTCGACCTGTTCGAATCGAAAAAGAAGCGCAACAACATCAAGCTGTACGTGCGCCGCGTCTTCATCATG $\begin{array}{llllllllllllllllllllllllll}\text { A } & P & F & D & L & F & E & S & K & K & K & R & N & N & I & K & L & Y & V & R & R & V & F & I & M\end{array}$ GATGACTGCGACGAGCTGATGCCGGATTGGCTGAACTTCGTGAAGGGCGTCGTCGACTCCGAAGACCTGCCGTTG $\begin{array}{lllllllllllllllllllllllllll}\mathrm{D} & \mathrm{D} & \mathrm{C} & \mathrm{D} & \mathrm{E} & \mathrm{L} & \mathrm{M} & \mathrm{P} & \mathrm{D} & \mathrm{W} & \mathrm{L} & \mathrm{N} & \mathrm{F} & \mathrm{V} & \mathrm{K} & \mathrm{G} & \mathrm{V} & \mathrm{V} & \mathrm{D} & \mathrm{S} & \mathrm{E} & \mathrm{D} & \mathrm{L} & \mathrm{P} & \mathrm{L}\end{array}$ AACATTTCCCGGGAGACTCTGCAGCAGAACAAGATCCTGCGCGTAATCAAGAAGAATTTGGTGAAGAAGTGCCTG $\begin{array}{lllllllllllllllllllllllllll}N & I & S & R & E & T & L & Q & Q & N & K & I & L & R & V & I & K & K & N & L & V & K & K & C & L\end{array}$ GAGATGTTCGCAGAGA TCGCGGAGAAGAACGATGATTACAAAAAGTTCTATGAGCAGTTCGGCAAGTGCTTGAAG $\begin{array}{lllllllllllllllllllllllllll}E & M & F & A & E & I & A & E & K & N & D & D & Y & K & K & F & Y & E & Q & F & G & K & C & L & K\end{array}$ TTGGGCGTGCACGAGGATTCCACGAACCGCACCAAGGTGGCCGAACTCATGCGCTACCAGACCTCGAAAACGGGC $\begin{array}{llllllllllllllllllllllllll}\mathrm{L} & \mathrm{G} & \mathrm{V} & \mathrm{H} & \mathrm{E} & \mathrm{D} & \mathrm{S} & \mathrm{T} & \mathrm{N} & \mathrm{R} & \mathrm{T} & \mathrm{K} & \mathrm{V} & \mathrm{A} & \mathrm{E} & \mathrm{L} & \mathrm{M} & \mathrm{R} & \mathrm{Y} & \mathrm{Q} & \mathrm{T} & \mathrm{S} & \mathrm{K} & \mathrm{T} & \mathrm{G}\end{array}$ GACGAATCCATCAGCTTGAAGGAGTACGTCGACAGGATGAAGGAGGGGCAGAATGACATCTACTACATCACTGGC $\begin{array}{lllllllllllllllllllllllll}D & E & S & I & S & L & K & E & Y & V & D & R & M & K & E & G & Q & N & D & I & Y & Y & I & T & G\end{array}$

GAAAGCATTGCCGCCGTGTCTTCTTCTCCGTTCCTGGAGACGCTGAGGAAGAAAGGGCTCGAGGTGTTGTACATG $\begin{array}{llllllllllllllllllllllllllllllllllll}E & S & I & A & A & V & S & S & S & P & F & L & E & T & L & R & K & K & G & L & E & V & L & Y & M\end{array}$ ACTGACCCGATCGATGAGTACTGCGTGCAACAGCTGAAAGAGTTCGATGGCAAAAAGTTGAAATCGACAACCAAG $\begin{array}{llllllllllllllllllllllllll}\mathrm{T} & \mathrm{D} & \mathrm{P} & \mathrm{I} & \mathrm{D} & \mathrm{E} & \mathrm{Y} & \mathrm{C} & \mathrm{V} & \mathrm{Q} & \mathrm{Q} & \mathrm{L} & \mathrm{K} & \mathrm{E} & \mathrm{F} & \mathrm{D} & \mathrm{G} & \mathrm{K} & \mathrm{K} & \mathrm{L} & \mathrm{K} & \mathrm{S} & \mathrm{T} & \mathrm{T} & \mathrm{K}\end{array}$ GAGGGCTTGGACATCGAGGACGAGGACGAAAAGAAAAAGCTGGAGGAGCAAAAGGCTGAGTTCGAGCCGCTCACC $\begin{array}{llllllllllllllllllllllllll}E & G & L & D & I & E & D & E & D & E & K & K & K & L & E & E & Q & K & A & E & F & E & P & L & T\end{array}$ AAGCTGATGAAGGAGGTCCTCGGCGACAAGGTGGAGAAGGTCATCATCAGCAGCCGCATGGCTGATTCGCCGTGC $\begin{array}{llllllllllllllllllllllllll}K & L & M & K & E & V & L & G & D & K & V & E & K & V & I & I & S & S & R & M & A & D & S & P & C\end{array}$ GTGCTGACCACGTCGGAGTACGGCTGGTCGGCGAACATGGAGCGCATCATGAAGGCCCAGGCCATGAGGGACAAC $\begin{array}{llllllllllllllllllllllllll}\mathrm{V} & \mathrm{L} & \mathrm{T} & \mathrm{T} & \mathrm{S} & \mathrm{E} & \mathrm{Y} & \mathrm{G} & \mathrm{W} & \mathrm{S} & \mathrm{A} & \mathrm{N} & \mathrm{M} & \mathrm{E} & \mathrm{R} & \mathrm{I} & \mathrm{M} & \mathrm{K} & \mathrm{A} & \mathrm{Q} & \mathrm{A} & \mathrm{M} & \mathrm{R} & \mathrm{D} & \mathrm{N}\end{array}$ TCGATGACA TCATACATGGTTTCCAAGAAGACCATGGAGGTCAACCCCAAGCATTCCATCATGTCCGAGCTGAGG $\begin{array}{llllllllllllllllllllllllll}\mathrm{S} & M & \mathrm{~T} & \mathrm{~S} & \mathrm{Y} & \mathrm{M} & \mathrm{V} & \mathrm{S} & \mathrm{K} & \mathrm{K} & \mathrm{T} & \mathrm{M} & \mathrm{E} & \mathrm{V} & \mathrm{N} & \mathrm{P} & \mathrm{K} & \mathrm{H} & \mathrm{S} & \mathrm{I} & \mathrm{M} & \mathrm{S} & \mathrm{E} & \mathrm{L} & \mathrm{R}\end{array}$

AAGAAGGCTTCGGCGGA TAAGTCGGACAAGACCGTGAAGGACTTGATCTGGTTGTTGTTCGACACCTCACTGCTC $\begin{array}{lllllllllllllllllllllllll}\mathrm{K} & \mathrm{K} & \mathrm{A} & \mathrm{S} & \mathrm{A} & \mathrm{D} & \mathrm{K} & \mathrm{S} & \mathrm{D} & \mathrm{K} & \mathrm{T} & \mathrm{V} & \mathrm{K} & \mathrm{D} & \mathrm{L} & \mathrm{I} & \mathrm{W} & \mathrm{L} & \mathrm{L} & \mathrm{F} & \mathrm{D} & \mathrm{T} & \mathrm{S} & \mathrm{L} & \mathrm{L}\end{array}$ ACCTCCGGCTTCAACCTGGACGAGCCCACGCAGTTCGCGGGCCGCATCCACCGCATGATCAAGCTCGGCCTGAGC $\begin{array}{llllllllllllllllllllllllllll}\mathrm{T} & \mathrm{S} & \mathrm{G} & \mathrm{F} & \mathrm{N} & \mathrm{L} & \mathrm{D} & \mathrm{E} & \mathrm{P} & \mathrm{T} & \mathrm{Q} & \mathrm{F} & \mathrm{A} & \mathrm{G} & \mathrm{R} & \mathrm{I} & \mathrm{H} & \mathrm{R} & \mathrm{M} & \mathrm{I} & \mathrm{K} & \mathrm{L} & \mathrm{G} & \mathrm{L} & \mathrm{S}\end{array}$ ATCGACGATGACGACGATGGCCTGGGCGACGACGACGACCTGCCGCCGCTTGAGGAGGTCGAGGGCGCCGCCGAT $\begin{array}{llllllllllllllllllllllllll}I & D & D & D & D & D & G & L & G & D & D & D & D & L & P & P & L & E & E & V & E & G & A & A & D\end{array}$ GAGGCTTCCAAGATGGAGGAGGTCGACTGAAGCAACGTGGGTTGCTTATCCGTGCATGCAAGAAACCACAGATGA $\begin{array}{lllllllllllllll} & \mathrm{A} & \mathrm{S} & \mathrm{K} & \mathrm{M} & \mathrm{E} & \mathrm{E} & \mathrm{V} & \mathrm{D} & \end{array}$

TGGCGTGTAGGCGCGCACATGACCGGCGCTAGTGCTGCGCGTGTCGCGATTGCATTGCTCCCGTCGATCACAAGA CTTGGACACCAGGCCGTTGTACAGGAATGCGCGGCAAAAAAAAAAAAAAAA

(a)

Figure 1: Continued. 


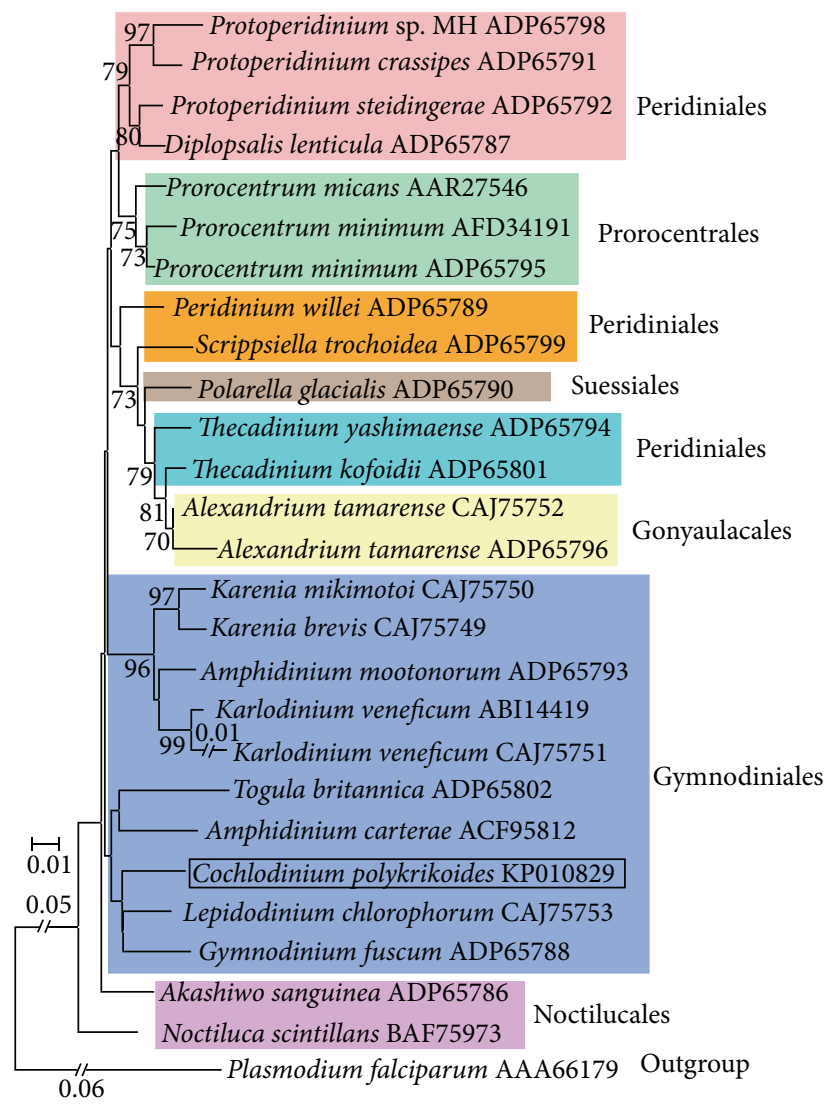

(b)

FIGURE 1: The cDNA and its predicted amino acid sequences of CPHSP90 and neighbor-joining tree of dinoflagellate HSP90s. (a) The cDNA and its predicted amino acid sequences: the conserved amino acids domains were marked in grey; the end domain "MEEVD" was boxed; (b) a neighbor-joining tree of dinoflagellate HSP90s. The phylogenetic tree was constructed in the MEGA 5 (bootstrap method with 1,000 replicates). The scale bar represents the number of amino acid substitutions per site. C. polykrikoides HSP90 was determined in the present study and marked in a box. Other dinoflagellate HSP90s and outgroup were obtained from NCBI database.

indicated that the CpHSP70 located in the cytoplasm of the cell [36].

A neighbor-joining tree was constructed using dinoflagellates HSP70 and other eukaryotic HSP70s (Figure 2(b)). As expected, all the dinoflagellate HSP70s were clustered into one clade, which showed closest relationship with Perkinsea, followed by Apicomplexa. All the analyzed dinoflagellates, Perkinsea, and Apicomplexa were grouped into one clade belonging to Alveolata.

\subsection{The Genomic Coding Structures of CpHSP70 and} CpHSP90. Genomic regions of each CpHSP70 and CpHSP90 were amplified by PCR. As a result, we found that no intron was presented in the CpHSP70 (KP010830) coding genome. In addition, the gene was encoded in tandem arrangement manner with the separation of intergenic spacers (Figures 3(a) and 3(b)), which was $397 \mathrm{bp}$ in length, and was found in CPHSP70 genome sequence. This result was similar to that of Amphidinium carterae [37]. On the other hand, interestingly, we found one intron as in CPHSP9O coding genome (KP010831) (Figure 3(c)), which was 454 bp in length, but no intergenic region was found, as judged by PCR. This structure was different from that of A. carterae HSP90 genomic sequence (25 introns), and A. carterae HSP90 gene was encoded in tandem arrangement. Although $A$. carterae HSP90 and CPHSP90 proteins showed highly homologous phylogenic relationships, their genomic DNA represented quite different characteristics. These results suggested that the same gene in different dinoflagellates displayed differential genome arrangement.

3.4. Effect of Algicide on CpHSP70 and CpHSP90 Transcription. Algicide chemical treatments are one of the powerful tools to remove HABs, and the physiological effects of individual algicides on the HABs have been widely investigated [38]. For example, the algicides oxidizing $\mathrm{NaOCl}$ and nonoxidizing $\mathrm{CuSO}_{4}$ can cause considerable decreases of $C$. polykrikoides cell numbers and pigment contents and also affect their chlorophyll autofluorescence [38]. Nevertheless, there is no report about the $\mathrm{CuSO}_{4}$ and $\mathrm{NaOCl}$ effect on the C. polykrikoides at molecular level, especially in terms of gene transcription.

In general, effects of the $\mathrm{CuSO}_{4}$ on aquatic organisms are relative to the formation of reactive oxygen species 
TTCCTCAATTTTGGCTCAAGTGTCAGTCATCCCCTATTCGTCTGTCCCCCTTTTCTCGGGGGGTTTTGCTTCGCC

1 ATGGCGAAAAAGACGCTGTTGGCATCGACTTGGGCACCACGTACAGCTGCGTGGGTGTATGGAAAAATAATGGC

$\begin{array}{lllllllllllllllllllllllllll}M & A & K & K & T & A & V & G & I & D & L & G & T & T & Y & S & C & V & G & V & W & K & N & N & G\end{array}$

76 GTTGAGATCATCGCCAACGACCAGGGCAACCGCACCACTCCCTCGTACGTGGGTTTCACGGACACCGAGCGCTTG $\begin{array}{llllllllllllllllllllllllll}\mathrm{V} & \mathrm{E} & \mathrm{I} & \mathrm{I} & \mathrm{A} & \mathrm{N} & \mathrm{D} & \mathrm{Q} & \mathrm{G} & \mathrm{N} & \mathrm{R} & \mathrm{T} & \mathrm{T} & \mathrm{P} & \mathrm{S} & \mathrm{Y} & \mathrm{V} & \mathrm{G} & \mathrm{F} & \mathrm{T} & \mathrm{D} & \mathrm{T} & \mathrm{E} & \mathrm{R} & \mathrm{L}\end{array}$

151 ATCGGCGACGCCGCGAAGAACCAGGTGGCCCGCAGCCCGGAGAACACCATTTTCGACGCCAAGCGCTTGATCGGC $\begin{array}{lllllllllllllllllllllllll}I & G & D & A & A & K & N & Q & V & A & R & S & P & E & N & T & I & F & D & A & K & R & L & I & G\end{array}$

226 CGCAAGTTCGCGGACCCCATCGTGCAGTCCGACATCAAGCTCTGGCCTTTCAAGGTCGTGGCAGGCCAGGGCGAC $\begin{array}{lllllllllllllllllllllllllll}R & \text { K } & \text { F } & \text { A } & \text { D } & \text { P } & \text { I } & \text { V } & \text { Q } & \text { S } & \text { D } & \text { I } & \text { K } & \text { L } & \text { W } & \text { P } & \text { F } & \text { K } & \text { V } & \text { V } & \text { A } & \text { G } & \text { Q } & \text { G } & \text { D }\end{array}$

301 AAGCCGATGATCGAGGTGAATGCCTCCGGCGAGGAAAAGCGCTTCCATCCCGAAGAGGTCTCTTCCATGATCTTG $\begin{array}{llllllllllllllllllllllllll}K & P & M & I & E & V & N & A & S & G & E & E & K & R & F & H & P & E & E & V & S & S & M & I & L\end{array}$

376 CTGAAGATGAAGGAGACGGCCGAGGCCTACTTGGGCACCAAGATCAACGATGCTGTGGTCACCGTGCCAGCGTAT $\begin{array}{lllllllllllllllllllllllll}\mathrm{L} & \mathrm{K} & \mathrm{M} & \mathrm{K} & \mathrm{E} & \mathrm{T} & \mathrm{A} & \mathrm{E} & \mathrm{A} & \mathrm{Y} & \mathrm{L} & \mathrm{G} & \mathrm{T} & \mathrm{K} & \mathrm{I} & \mathrm{N} & \mathrm{D} & \mathrm{A} & \mathrm{V} & \mathrm{V} & \mathrm{T} & \mathrm{V} & \mathrm{P} & \mathrm{A} & \mathrm{Y}\end{array}$

451 TTCAACGACTCCCAGCGCCAGGCCACCAAGGACGCCGGCACCATCTCCGGCATGAATGTGCTCCGCATCATCAAC $\begin{array}{llllllllllllllllllllllllll}F & N & D & S & Q & R & Q & A & T & K & D & A & G & T & I & S & G & M & N & V & \text { L } & R & I & I & N\end{array}$

526 GAGCCCACCGCTGCCGCCATTGCTTACGGCTTGGACAAGAAGGGCACCGGTGAGCGCAACATCCTCATCTACGAC

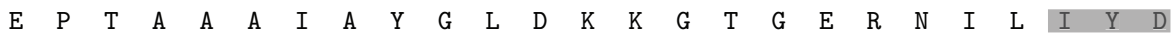

601 ATGGGTGGCGGCACTTTCGACGTGTCTTTGCTGACCATCGAGGACGGAATCTTCGAGGTGAAGGCCACGGCCGGC $\begin{array}{llllllllllllllllllllllllllllllll}M & G & G & G & T & F & D & V & S & L & L & T & I & E & D & G & I & F & E & V & K & A & T & A & G\end{array}$

676 GACACCCACTTGGGTGGTGAGGATTTCGACAATCGTATAGTGGATTTCTGCTTGCAGGACTTCAAGCGCAAGAAC $\begin{array}{lllllllllllllllllllllllll}\text { D } & \text { T } & \text { H } & \text { L } & G & G & \text { E } & \text { D } & \text { F } & \text { D } & \text { N } & \text { R } & \text { I } & \text { V } & \text { D } & \text { F } & \text { C } & \text { L } & \text { Q } & \text { D } & \text { F } & \text { K } & \text { R } & \text { K } & N\end{array}$

751 CGCGGCAAGGACATGTCCGGCAACCACCGCGCAATTCGCCGCCTGAGGACGCAGTGCGAGCGCGCTAAGCGGACC $\begin{array}{llllllllllllllllllllllllll}R & G & K & D & M & S & G & N & H & R & A & I & R & R & L & R & T & Q & C & E & R & A & K & R & T\end{array}$

826 TTGTCTTCCTCAACACAGGCCACGATCGAGATCGACTCTCTGTTCGAAGGCATCGATTTCTCGTGCCCGCTGTCC $\begin{array}{llllllllllllllllllllllllll}\mathrm{L} & \mathrm{S} & \mathrm{S} & \mathrm{S} & \mathrm{T} & \mathrm{Q} & \mathrm{A} & \mathrm{T} & \mathrm{I} & \mathrm{E} & \mathrm{I} & \mathrm{D} & \mathrm{S} & \mathrm{L} & \mathrm{F} & \mathrm{E} & \mathrm{G} & \mathrm{I} & \mathrm{D} & \mathrm{F} & \mathrm{S} & \mathrm{C} & \mathrm{P} & \mathrm{L} & \mathrm{S}\end{array}$

901 CGCGCGCGTTTCGAAGAGCTGAACATGGACTATTTCCGCAATTCCATGGGCCCCGTGGAGAAGTGCCTGCGCGAC $\begin{array}{lllllllllllllllllllllllllll}R & A & R & F & E & E & L & N & M & D & Y & F & R & N & S & M & G & P & V & E & K & C & L & R & D\end{array}$

976 AGCGGCATCGACAAGCGGAACGTGCACGAGGTGGTGCTGGTGGGCGGA TCCACACGCATCCCCAAGGTTCAGTCG $\begin{array}{llllllllllllllllllllllllll}S & G & I & D & K & R & N & V & H & E & V & V & L & V & G & G & S & T & R & I & P & K & V & Q & S\end{array}$

1051 ATGATTCAGGAGTTCTTCAACGGCAAGGAGCCGTGCAAATCCATCAACCCCGACGAGGCTGTTGCTTTTGGAGCA $\begin{array}{lllllllllllllllllllllllll}M & I & Q & E & F & F & N & G & K & E & P & C & K & S & I & N & P & D & E & A & V & A & F & G & A\end{array}$

1126 GCGGTGCAGGCCGCCATCTTGACAGGCGAGGGCTCGTCACAGGTGCAGGACCTGCTGCTTTTGGATGTGACGCCT $\begin{array}{lllllllllllllllllllllllll}\text { A } & \text { V } & \text { Q } & \text { A } & \text { A } & \text { I } & \text { L } & \text { T } & G & \text { E } & \text { G } & \text { S } & \text { S } & \text { Q } & \text { V } & \text { Q } & \text { D } & \text { L } & \text { L } & \text { L } & \text { L } & \text { D } & \text { V } & \text { T } & \text { P }\end{array}$

1201 TTGTCCATGGGTCTCGAGACGGCTGGTGGTGTGATGACCAAGCTCATCGAGAGGAACACGACGATCCCCACCAAA $\begin{array}{lllllllllllllllllllllllllll}\text { L } & S & M & G & \text { L } & E & \text { T } & \text { A } & G & G & \text { V } & \text { M } & \text { T } & \text { K } & \text { L } & \text { I } & E & R & N & T & T & \text { I } & P & \text { T } & \text { K }\end{array}$

1276 AAGGGGCAGACCTTCACGACGTATGCAGACAACCAGCCTGGTGTGCTGATCCAGGTGTTCGAGGGCGAGCGAGCG $\begin{array}{llllllllllllllllllllllllll}K & G & Q & T & F & T & T & Y & A & D & N & Q & P & G & V & L & I & Q & V & F & E & G & E & R & A\end{array}$

1351 ATGACGAAGGACAACAACTTGTTGGGGAAGTTCCACTTGGACGGCATTCCTCCGGCCCCCCGTGGCGTGCCGCAG $\begin{array}{lllllllllllllllllllllllll}M & \mathrm{~T} & \mathrm{~K} & \mathrm{D} & \mathrm{N} & \mathrm{N} & \mathrm{L} & \mathrm{L} & \mathrm{G} & \mathrm{K} & \mathrm{F} & \mathrm{H} & \mathrm{L} & \mathrm{D} & \mathrm{G} & \mathrm{I} & \mathrm{P} & \mathrm{P} & \mathrm{A} & \mathrm{P} & \mathrm{R} & \mathrm{G} & \mathrm{V} & \mathrm{P} & \mathrm{Q}\end{array}$

1426 ATTGAGGTCACTTTCGACATTGACGCCAACGGCATCCTGAACGTGTCCGCCCAGGACAAGTCCACGGGCAAGAGC $\begin{array}{lllllllllllllllllllllllll}I & E & V & T & F & D & I & D & A & N & G & I & L & N & V & S & A & Q & D & K & S & T & G & K & S\end{array}$

1501 AATCAGATCACCATCACGAACGAGAAGGGTCGGTTGTCTCAGTCGGAAATCGACCGCATGGTGCAGGAGGCCGAG $\begin{array}{llllllllllllllllllllllllll}N & Q & I & T & I & T & N & E & K & G & R & L & S & Q & S & E & I & D & R & M & V & Q & E & A & E\end{array}$

1576 AAGTACCGTGCGGAGGACGAGGCCAACCGTGTGAAGATTGAGGCGAAAAACGGCTTGGAGAACTACTGCTTCACT $\begin{array}{lllllllllllllllllllllllllll}K & Y & R & A & E & D & E & A & N & R & V & K & I & E & A & K & N & G & L & E & N & Y & C & F & T\end{array}$

1651 ATGCGTAACACGCTGCAGGAGGAGAAGTTGAAGGACAAGTTCGAGTCCGGTGACAAGGAGAAGATTGAGCAGGCT $\begin{array}{lllllllllllllllllllllllll}M & R & N & T & L & Q & E & E & K & L & K & D & K & F & E & S & G & D & K & E & K & I & E & Q & A\end{array}$

1726 GTGCAAGACACCTTGGATTGGTTAGACAAGAACCAGCTGGCCGAGAAGGACGAGTTCGAGGCCAAGCAGAAAGAG $\begin{array}{lllllllllllllllllllllllll}V & Q & D & T & L & D & W & L & D & K & N & Q & L & A & E & K & D & E & F & E & A & K & Q & K & E\end{array}$

1801 TTGGAGGGCGTTGTCAATCCCATCATGATGAAGGTCTACCAGGCCGCCGGAGGAGGTGGCATGCCTGAGGGTGGC

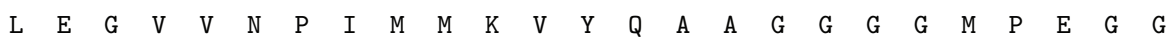

1876 ATGCCAGGAGGAGGCTTTGGCGGCGCTGCCCCGGGAGGGGCAGGAGGCCCCACCGTGGAGGAGGTCGACTAGATG

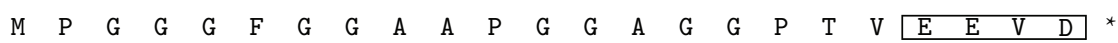

1951 GTTTTCCCTCATGACCTGAGGATTCGCCGAGTGTGCACGTGCTCAATTCAGCAACCCCAGACTGTCCCACAAAA 2026 AAAAAAAAAAAA

(a)

Figure 2: Continued. 


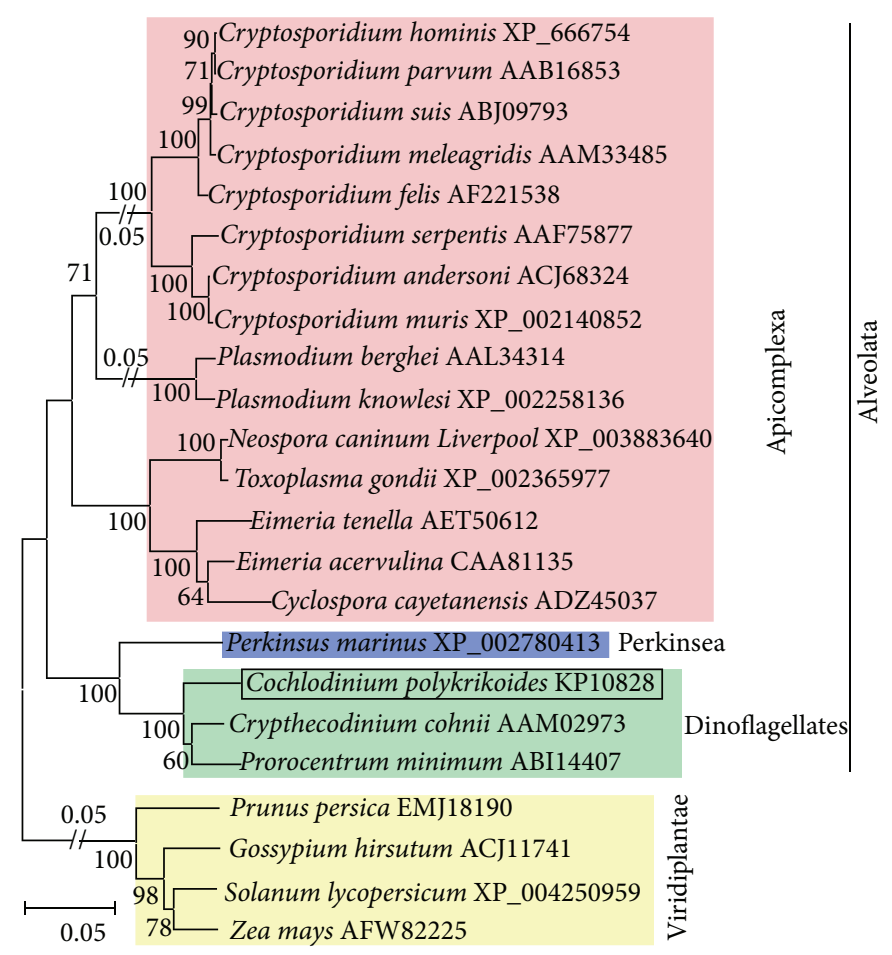

(b)

FIGURE 2: The cDNA and its predicted amino acid sequences of CpHSP70 and neighbor-joining tree of dinoflagellate HSP70s. (a) The cDNA and its predicted amino acid sequences: the conserved amino acids domains were marked in grey; the end domain "EEVD" was boxed; (b) the phylogenetic tree was constructed in the MEGA 5 (bootstrap method with 1,000 replicates), and the scale bar represents the number of amino acid substitutions per site. CPHSP70 was determined in the present study and marked in a box. Other dinoflagellates HSP70s and outgroup were obtained from NCBI database.

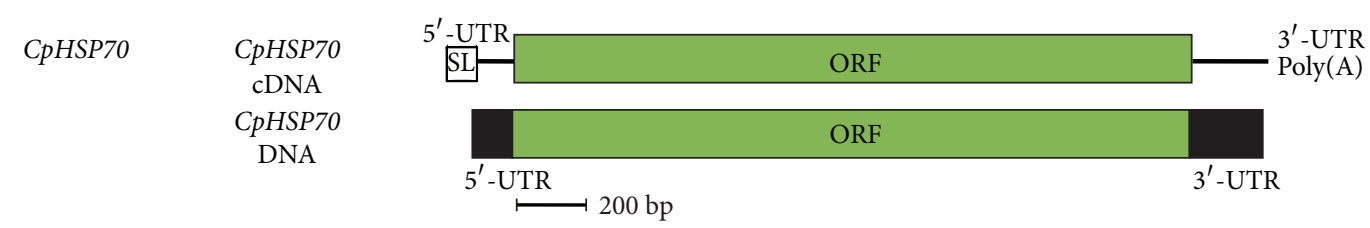

(a)

\begin{tabular}{|c|c|c|c|c|c|c|c|c|c|}
\hline & Copy 1 & $\begin{array}{l}\text { Intergenic } \\
\text { region } 1\end{array}$ & Copy 2 & $\begin{array}{c}\text { Intergenic } \\
\text { region } 2\end{array}$ & Copy 3 & $\begin{array}{l}\text { Intergenic } \\
\text { region } 3\end{array}$ & Copy 4 & $\begin{array}{c}\text { Intergenic } \\
\text { region } 4\end{array}$ & Copy 5 \\
\hline CрHSP70 & & & & & & & & & \\
\hline
\end{tabular}

(b)

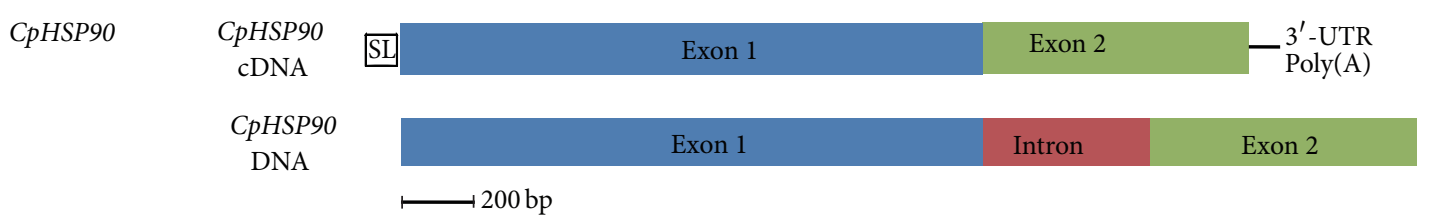

(c)

FIguRE 3: Schematic structures of genomic DNA of CPHSP70 and CpHSP90. (a) Comparison of DNA and cDNA of CPHSP70. (b) Predicted genomic DNA structure of the CPHSP70: the different copies of the CPHSP70 genes are separated by intergenic regions. (c) Comparison of DNA and cDNA of CPHSP90. One intron (red box) was found in the genomic DNA sequence of CpHSP90. 


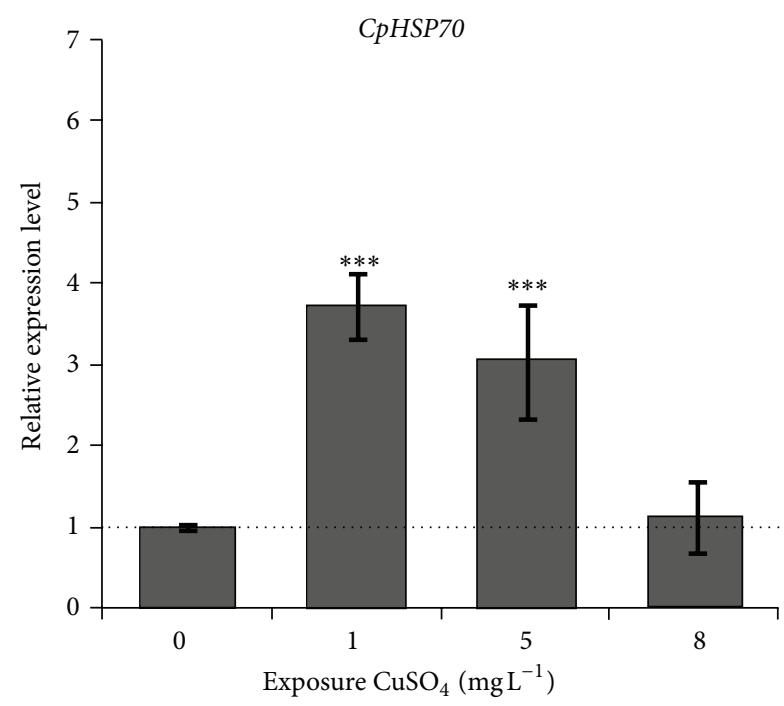

(a)

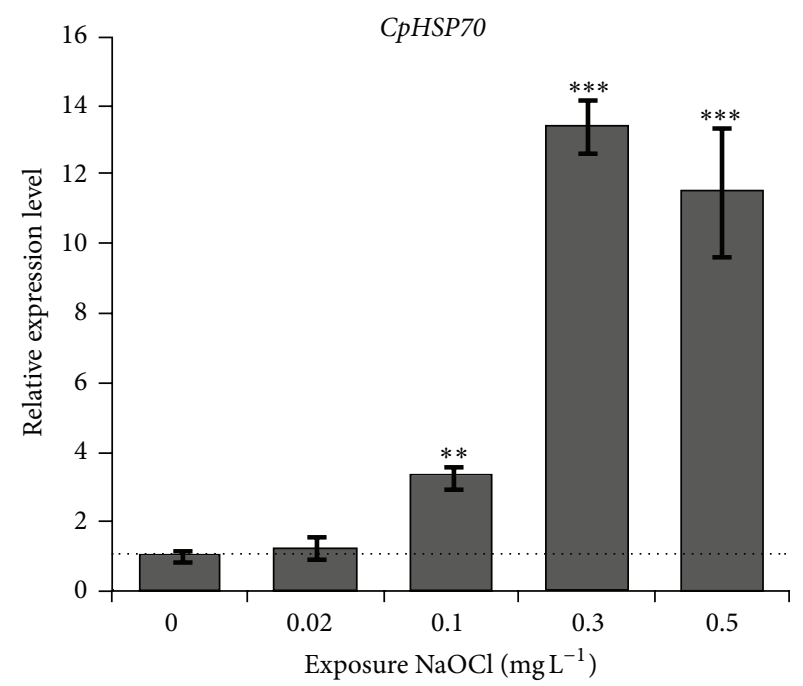

(c)

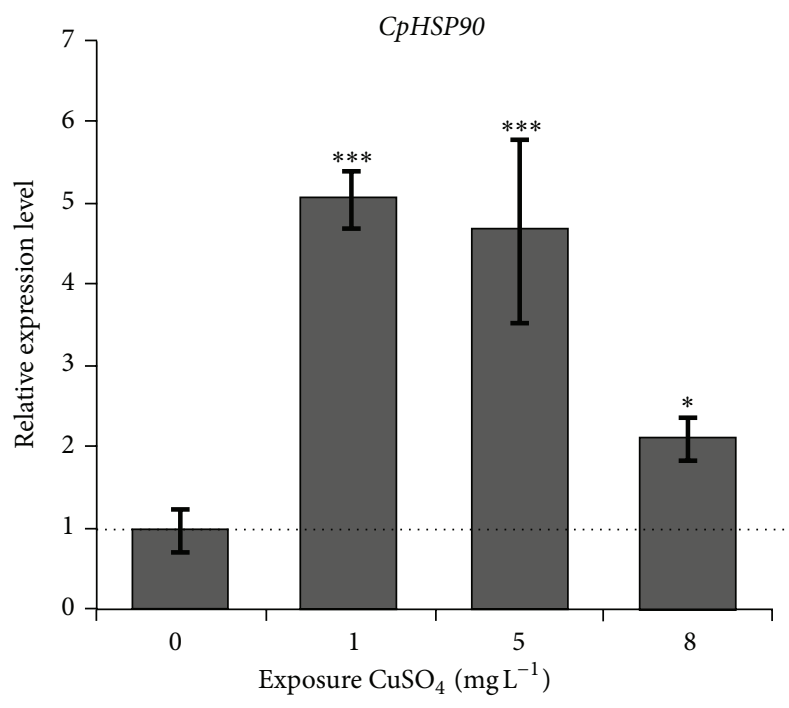

(b)

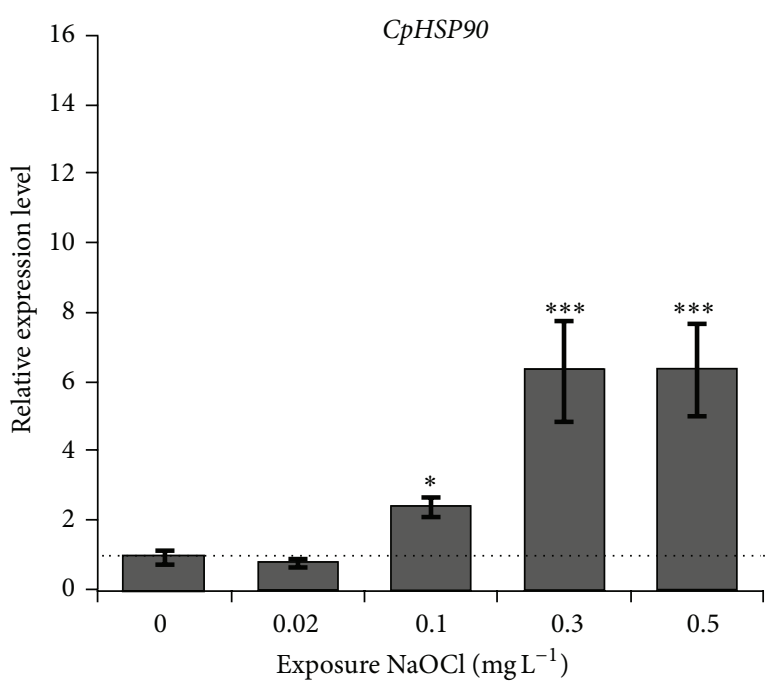

(d)

Figure 4: The gene expression profile of $\mathrm{CpHSP70}$ and $\mathrm{CpHSP90}$ after $\mathrm{CuSO}_{4}$ and $\mathrm{NaOCl}$ treatment, respectively. (a, b) Total RNA was extracted from C. polykrikoides at $24 \mathrm{~h}$ after treatment with $1 \mathrm{mg} \mathrm{L}^{-1}, 5 \mathrm{mg} \mathrm{L}^{-1}$, and $8 \mathrm{mg} \mathrm{L}^{-1}$ of $\mathrm{CuSO}_{4}$, respectively. (c, d) Total RNA was extracted from C. polykrikoides after treatment with $0.02 \mathrm{mg} \mathrm{L}^{-1}, 0.10 \mathrm{mg} \mathrm{L}^{-1}, 0.3 \mathrm{mg} / \mathrm{L}$, and $0.5 \mathrm{mg} \mathrm{L}^{-1} \mathrm{NaOCl}$, respectively. The $18 S \mathrm{rRNA}$ gene was used as the internal control to normalize the amount of templates in qRT-PCR. Results are given as the means of triplicate \pm SD. The significant differences between the treated group and the control group are highlighted by one-way ANOVA; ${ }^{*} P<0.05,{ }^{* *} P<0.01$, ${ }^{* * *} P<0.001$.

(ROS) and can regulate the photosynthesis related gene expression and increase the antioxidant enzyme activity in the algae $[39,40]$. In the present study, the transcriptional expression of CPHSP90 and CpHSP70 showed similar expression pattern after $\mathrm{CuSO}_{4}$ treatment (Figure 4). The transcriptional expression level of these two genes was first upregulated and then decreased with increased concentration of $\mathrm{CuSO}_{4}$. The CpHSP90 transcription showed similar expression pattern under $1.0 \mathrm{mg} \mathrm{L}^{-1}$ and $5.0 \mathrm{mg} \mathrm{L}^{-1} \mathrm{CuSO}_{4}$ treatments with 5.1- and 4.7-fold changes compared to that of control, respectively. These expression patterns were also displayed by CPHSP70, showing 3.7- and 3.1-fold changes under $1.0 \mathrm{mg} \mathrm{L}^{-1}$ and $5.0 \mathrm{mg} \mathrm{L}^{-1} \mathrm{CuSO}_{4}$ treatments compared to that of control. Either CpHSP90 or CpHSP70 was distinctly decreased compared to those of $1.0 \mathrm{mg} \mathrm{L}^{-1}$ or $5.0 \mathrm{mg} \mathrm{L}^{-1}$ $\mathrm{CuSO}_{4}$ treatment (Figures $4(\mathrm{a})$ and $4(\mathrm{~b})$ ). This result suggested that the CPHSP90 and CPHSP70 may be involved in $\mathrm{CuSO}_{4}$ induced gene regulation in C. polykrikoides. This result was congruent with our previous results, showing that both genes were considerably induced by exposure of $\mathrm{CuSO}_{4}$ in the dinoflagellate P. minimum $[27,28]$. However, we found that the expression patterns of HSP70 genes were different between C. polykrikoides and P. minimum (Pm) exposed to the same $\mathrm{CuSO}_{4}$. The CPHSP70 expression was increased 


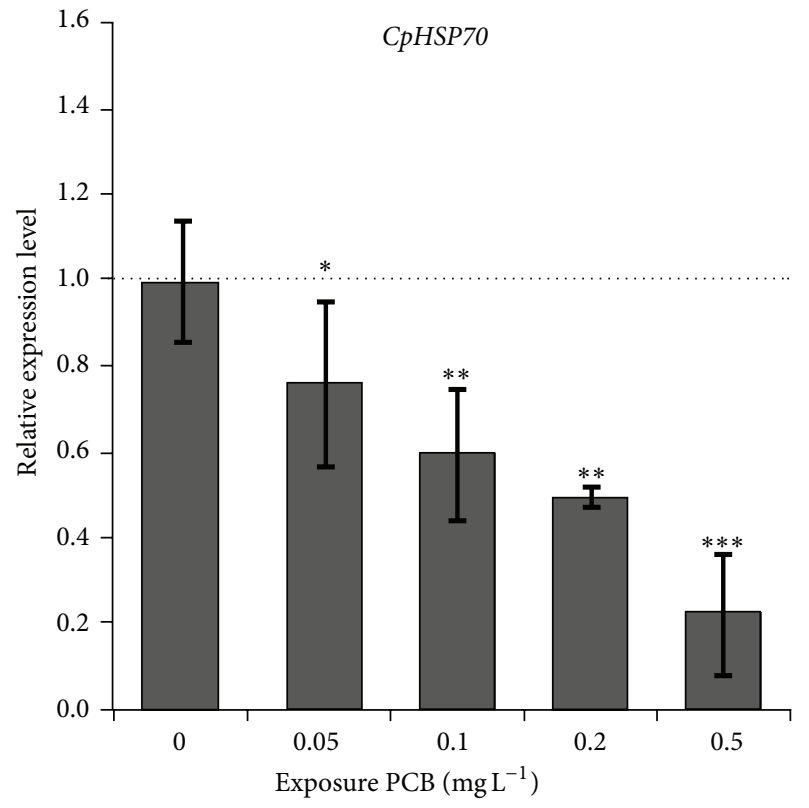

(a)

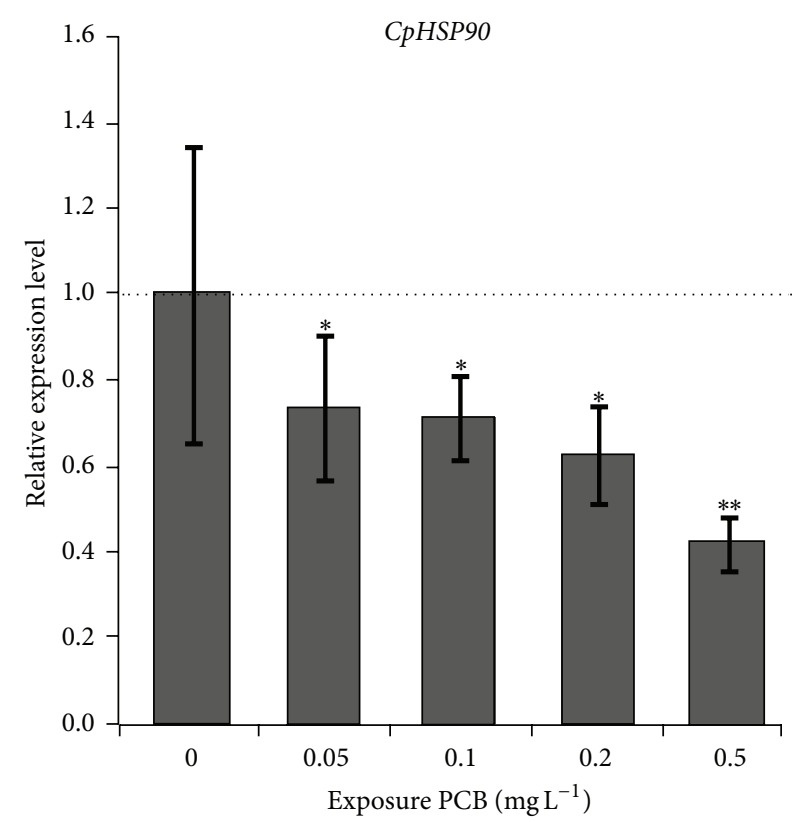

(b)

FIGURE 5: Gene expression profile of CPHSP70 (a) and CPHSP90 (b) after PCB treatment, respectively. Total RNA was extracted from C. polykrikoides at $24 \mathrm{~h}$ after treatment with $0.05 \mathrm{mg} \mathrm{L}^{-1}, 0.1 \mathrm{mg} \mathrm{L}^{-1}, 0.2 \mathrm{mg} \mathrm{L}^{-1}$, and $0.5 \mathrm{mg} \mathrm{L}^{-1}$ of PCB, respectively. The $18 S r R N A$ gene was used as the internal control to normalize the amount of templates in qRT-PCR. Results are given as the means of triplicate \pm SD. The significant differences between the treated group and the control group are highlighted by one-way ANOVA; ${ }^{* *} P<0.01,{ }^{* * *} P<0.001$.

gradually till high dose $\left(8.0 \mathrm{mg} \mathrm{L}^{-1}\right.$ of $\left.\mathrm{CuSO}_{4}\right)$, whereas the PmHSP70 expression was the highest level at $1.0 \mathrm{mg} \mathrm{L}^{-1}$ and then decreased, possibly due to cell deaths. According to these results, we predicted that the HSP90 and HSP70 are commonly involved in metal $\mathrm{CuSO}_{4}$ induced gene regulation in the dinoflagellates, but their expression patterns may depend on exposed doses and testing species.

Oxidizing chlorine is one of biocides that are commonly used in controlling the quality of the water. It can damage the cell by producing ROS. Hypochlorites may have primary deleterious effect on the DNA synthesis or progress oxidation of thiol groups and further effect on the cell wall and protein [41]. In the present study, the expression pattern showed response of $C P H S P 90$ and $C p H S P 70$ depending on the doses of the $\mathrm{NaOCl}$. Interestingly, CpHSP9O and CpHSP70 were not induced by lower concentration $\left(0.02 \mathrm{~m} \mathrm{~L}^{-1}\right)$ of $\mathrm{NaOCl}$ but significantly upregulated by relatively high concentrations $\left(0.1,0.3\right.$, and $0.5 \mathrm{mg} \mathrm{L}^{-1}$ ) of $\mathrm{NaOCl}$ (Figures 4 (c) and 4(d)). Both CPHSP90 and CPHSP70 in $0.3 \mathrm{mg} \mathrm{L}^{-1} \mathrm{NaOCl}$ treated cells showed 8.5- and 13.4-fold changes compared to that of control, which were highest expression level among $\mathrm{NaOCl}$ treated cells. The chlorine-based disinfectants induced HSP70 gene expression was also found in the $C$. parvum oocysts [42].

\subsection{Effect of PCB on CPHSP70 and CpHSP90 Transcription.} There are many of chemicals that presented in the aquatic system by industry or agriculture sewerage. PCBs are used in the industry and commonly present in aquatic ecosystems. They are one of the endocrine disturbing chemicals (EDCs) and have toxic effect to various organisms [43]. According to our previous work [44], EDCs, including PCB, were very toxic to microalgae, potentially affecting the photosystem II energy flow, of which results suggested their toxic effect on the dinoflagellates.

In this study, we examined the gene expressional response of CPHSP70 and CPHSP90 in C. polykrikoides exposed to $\mathrm{PCB}$. The transcriptional expressions of CPHSP70 and CPHSP90 were gradually decreased with increasing $\mathrm{PCB}$ concentration (Figure 5), with lowest expression level of 0.42 - and 0.23 -fold under $0.5 \mathrm{mg} \mathrm{L}^{-1} \mathrm{PCB}$ exposure compared to untreated cultures. However, neither PmHSP90 nor PmHSP70 was up- or downregulated by the PCB exposure in the dinoflagellate Prorocentrum minimums [27, 28]. According to these results, we speculated that the PCB may have a differential effect on the dinoflagellates among species. The aroclor 1016, which is one of dioxin-like PCBs, was employed. It is clear that the aryl hydrocarbon receptor (Ahr) mediated pathway is involved in the dioxin-like PCBs adverse effect mechanism [45, 46]. A dimer of HSP90 is essential compound that binds to the inactive Ahr protein in the cytoplasm and needs to be released from Ahr protein complex when Ahr is activated [47-50]. According to these findings, we predict that the CpHSP90 may bind to Ahr and express at a high level in the normal conditions. The CPHSP9O may be released, and then the protein may be activated to participate in the signal transduction of the cells, when C. polykrikoides is exposed to PCB. The essential CpHSP90 amount was decreased with increasing concentration of $\mathrm{PCB}$ in the C. polykrikoides. At present, it was not clear; thus we needed more experiments to clarify these gene responses 
in the dinoflagellate, by using additional EDCs and testing species in future.

In addition to this, the CPHSP70 and CPHSP9O showed similar expression patterns under the same PCB treatment (Figures 4 and 5). Similar results were found in the benzo[a]pyrene treated clam Ruditapes philippinarum [51]. Both CPHSP9O and CPHSP70 proteins contain EEVD motif, which is tetratricopeptide repeat domain binding site at the C-terminus, and some proteins can bind with HSP90 and HSP70 to assemble as protein complex to play function such as in the triage of damaged and aberrant proteins for degradation process $[16,52]$. The gene transcription pattern of CPHSP9O and CPHSP70 results implied these two genes may have cooperative function in the C. polykrikoides toxicant induced gene regulation $[16,52]$.

In conclusion, this study firstly determined full length cDNAs of two HSPs (HSP70/90) from the harmful dinoflagellate C. polykrikoides and characterized molecular features such as conserved motifs, coding genomic region, and phylogenetic relatedness to other eukaryotes. CPHSP70 had quite similar cDNA and genomic coding structures (e.g., no intron and tandem arrangement) to those in the other dinoflagellates; however, CPHSP90 was different from those of other dinoflagellate HSP90s in coding genomic structure (one intron and no intergenic region). These suggested their homologous functions with difference of genomic DNA evolutionary events. In addition, both CPHSP90 and CPHSP70 may be involved in responding to the $\mathrm{CuSO}_{4}, \mathrm{NaOCl}$, and PCB caused stress.

\section{Conflict of Interests}

The authors declare that there is no conflict of interests regarding the publication of this paper.

\section{Acknowledgments}

This work was supported by the National Research Foundation of Korea grant funded by the Korean Government (NRFM1A5A1-2013-044476 and 2013R1A1A 2013596) and by a grant from the National Fisheries Research and Development (NFRDI) funded to J.-S. Ki.

\section{References}

[1] D. M. Anderson, "The ecology and oceanography of harmful algal blooms: multidisciplinary approaches to research and management," IOC Technical Series 74, IOC/2007/TS/74, UNESCO, 2007.

[2] Y. S. Lee and S. Y. Lee, "Factors affecting outbreaks of Cochlodinium polykrikoides blooms in coastal areas of Korea," Marine Pollution Bulletin, vol. 52, no. 6, pp. 626-634, 2006.

[3] R. M. Kudela and C. J. Gobler, "Harmful dinoflagellate blooms caused by Cochlodinium sp.: global expansion and ecological strategies facilitating bloom formation," Harmful Algae, vol. 14, pp. 71-86, 2012.

[4] R. V. Azanza, L. T. David, R. T. Borja, I. U. Baula, and Y. Fukuyo, "An extensive Cochlodinium bloom along the western coast of
Palawan, Philippines," Harmful Algae, vol. 7, no. 3, pp. 324-330, 2008.

[5] C. J. Gobler, D. L. Berry, O. R. Anderson et al., "Characterization, dynamics, and ecological impacts of harmful Cochlodinium polykrikoides blooms on eastern Long Island, NY, USA," Harmful Algae, vol. 7, no. 3, pp. 293-307, 2008.

[6] Y. Z. Tang and C. J. Gobler, "Cochlodinium polykrikoides blooms and clonal isolates from the northwest Atlantic coast cause rapid mortality in larvae of multiple bivalve species," Marine Biology, vol. 156, no. 12, pp. 2601-2611, 2009.

[7] Y. S. Lee, "Factors affecting outbreaks of high-density Cochlodinium polykrikoides red tides in the coastal seawaters around Yeosu and Tongyeong, Korea," Marine Pollution Bulletin, vol. 52, no. 10, pp. 1249-1259, 2006.

[8] D. Kim, T. Oda, T. Muramatsu, Y. Matsuyama, and T. Honjo, "Possible factors responsible for the toxicity of Cochlodinium polykrikoides, a red tide phytoplankton," Comparative Biochemistry and Physiology C: Toxicology \& Pharmacology, vol. 132, no. 4, pp. 415-423, 2002.

[9] S. J. Oh, Y. H. Yoon, D.-I. Kim, Y. Shimasaki, Y. Oshima, and T. Honjo, "Effect of light quantity and quality on the growth of the harmful dinoflagellate, Cochlodinium polykrikoides Margalef (Dinophyceae)," ALGAE, vol. 21, no. 3, pp. 311-316, 2006.

[10] G. Onitsuka, K. Miyahara, N. Hirose et al., "Large-scale transport of Cochlodinium polykrikoides blooms by the Tsushima Warm Current in the southwest Sea of Japan," Harmful Algae, vol. 9, no. 4, pp. 390-397, 2010.

[11] M. O. Lee, J. H. Choi, and I. H. Park, "Outbreak conditions for Cochlodinium polykrikoides blooms in the southern coastal waters of Korea," Marine Environmental Research, vol. 70, no. 2, pp. 227-238, 2010.

[12] S. C. Gupta, A. Sharma, M. Mishra, R. K. Mishra, and D. K. Chowdhuri, "Heat shock proteins in toxicology: how close and how far?” Life Sciences, vol. 86, no. 11-12, pp. 377-384, 2010.

[13] J. G. Sørensen, T. N. Kristensen, and V. Loeschcke, "The evolutionary and ecological role of heat shock proteins," Ecology Letters, vol. 6, no. 11, pp. 1025-1037, 2003.

[14] R. Li, G.-Z. Chen, N. F. Y. Tam et al., "Toxicity of bisphenol A and its bioaccumulation and removal by a marine microalga Stephanodiscus hantzschii," Ecotoxicology and Environmental Safety, vol. 72, no. 2, pp. 321-328, 2009.

[15] M. Morales, R. Planelló, P. Martínez-Paz et al., "Characterization of $H s p 70$ gene in Chironomus riparius: expression in response to endocrine disrupting pollutants as a marker of ecotoxicological stress," Comparative Biochemistry and Physiology C: Toxicology and Pharmacology, vol. 153, no. 1, pp. 150-158, 2011.

[16] W. B. Pratt, Y. Morishima, H.-M. Peng, and Y. Osawa, "Proposal for a role of the Hsp90/Hsp70-based chaperone machinery in making triage decisions when proteins undergo oxidative and toxic damage," Experimental Biology and Medicine, vol. 235, no. 3, pp. 278-289, 2010.

[17] I. Mukhopadhyay, A. Nazir, D. K. Saxena, and D. Kar Chowdhuri, "Heat shock response: $h s p 70$ in environmental monitoring," Journal of Biochemical and Molecular Toxicology, vol. 17, no. 5, pp. 249-254, 2003.

[18] M. A. Torres, M. P. Barros, S. C. G. Campos et al., "Biochemical biomarkers in algae and marine pollution: a review," Ecotoxicology and Environmental Safety, vol. 71, no. 1, pp. 1-15, 2008. 
[19] B. F. Donnelly, P. G. Needham, A. C. Snyder et al., "Hsp70 and Hsp90 multichaperone complexes sequentially regulate thiazide-sensitive cotransporter endoplasmic reticulumassociated degradation and biogenesis," The Journal of Biological Chemistry, vol. 288, no. 18, pp. 13124-13135, 2013.

[20] J. B. French, H. Zhao, S. An et al., "Hsp70/Hsp90 chaperone machinery is involved in the assembly of the purinosome," Proceedings of the National Academy of Sciences of the United States of America, vol. 110, no. 7, pp. 2528-2533, 2013.

[21] M. P. Mayer and B. Bukau, "Hsp70 chaperones: Cellular functions and molecular mechanism," Cellular and Molecular Life Sciences, vol. 62, no. 6, pp. 670-684, 2005.

[22] N. M. Fast, L. Xue, S. Bingham, and P. J. Keeling, "Reexamining alveolate evolution using multiple protein molecular phylogenies," Journal of Eukaryotic Microbiology, vol. 49, no. 1, pp. 30-37, 2002.

[23] K. Shalchian-Tabrizi, M. A. Minge, T. Cavalier-Smith, J. M. Nedreklepp, D. Klaveness, and K. S. Jakobsen, "Combined heat shock protein 90 and ribosomal RNA sequence phylogeny supports multiple replacements of dinoflagellate plastids," The Journal of Eukaryotic Microbiology, vol. 53, no. 3, pp. 217-224, 2006.

[24] M. Hoppenrath and B. S. Leander, "Dinoflagellate phylogeny as inferred from heat shock protein 90 and ribosomal gene sequences," PLoS ONE, vol. 5, no. 10, Article ID el3220, 2010.

[25] H. Zhang, D. A. Campbell, N. R. Sturm, C. F. Dungan, and S. Lin, "Spliced leader RNAs, mitochondrial gene frameshifts and multi-protein phylogeny expand support for the genus Perkinsus as a unique group of alveolates," PLoS ONE, vol. 6, no. 5, Article ID e19933, 2011.

[26] N. N. Rosic, M. Pernice, S. Dove, S. Dunn, and O. HoeghGuldberg, "Gene expression profiles of cytosolic heat shock proteins Hsp70 and Hsp90 from symbiotic dinoflagellates in response to thermal stress: possible implications for coral bleaching," Cell Stress and Chaperones, vol. 16, no. 1, pp. 69-80, 2011.

[27] R. Guo, V. Ebenezer, and J.-S. Ki, “Transcriptional responses of heat shock protein 70 (Hsp70) to thermal, bisphenol A, and copper stresses in the dinoflagellate Prorocentrum minimum," Chemosphere, vol. 89, no. 5, pp. 512-520, 2012.

[28] R. Guo and J.-S. Ki, "Differential transcription of heat shock protein 90 (HSP90) in the dinoflagellate Prorocentrum minimum by copper and endocrine-disrupting chemicals," Ecotoxicology, vol. 21, no. 5, pp. 1448-1457, 2012.

[29] M. G. Murray and W. F. Thompson, "Rapid isolation of high molecular weight plant DNA," Nucleic Acids Research, vol. 8, no. 19, pp. 4321-4326, 1980.

[30] K. Tamura, D. Peterson, N. Peterson, G. Stecher, M. Nei, and S. Kumar, "MEGA5: molecular evolutionary genetics analysis using maximum likelihood, evolutionary distance, and maximum parsimony methods," Molecular Biology \& Evolution, vol. 28, no. 10, pp. 2731-2739, 2011.

[31] N. Saitou and M. Nei, "The neighbor-joining method: a new method for reconstructing phylogenetic trees," Molecular Biology and Evolution, vol. 4, no. 4, pp. 406-425, 1987.

[32] J. Felsenstein, "Confidence limits on phylogenies: an approach using the bootstrap," Evolution, vol. 39, no. 4, pp. 783-791, 1985.

[33] D. T. Jones, W. R. Taylor, and J. M. Thornton, "The rapid generation of mutation data matrices from protein sequences," Computer Applications in the Biosciences, vol. 8, no. 3, pp. 275282, 1992.
[34] Q. Gao, L. Song, D. Ni, L. Wu, H. Zhang, and Y. Chang, "CDNA cloning and mRNA expression of heat shock protein 90 gene in the haemocytes of Zhikong scallop Chlamys farreri," Comparative Biochemistry and Physiology B: Biochemistry and Molecular Biology, vol. 147, no. 4, pp. 704-715, 2007.

[35] H.-J. Ahn, S. Kim, and H.-W. Nam, "Molecular cloning of the $82-\mathrm{kDa}$ heat shock protein (HSP90) of Toxoplasma gondii associated with the entry into and growth in host cells," Biochemical and Biophysical Research Communications, vol. 311, no. 3, pp. 654-659, 2003.

[36] C. Scheufler, A. Brinker, G. Bourenkov et al., "Structure of TPR domain-peptide complexes: critical elements in the assembly of the Hsp70-Hsp90 multichaperone machine," Cell, vol. 101, no. 2, pp. 199-210, 2000.

[37] T. R. Bachvaroff and A. R. Place, "From stop to start: Tandem gene arrangement, copy number and Trans-splicing sites in the dinoflagellate Amphidinium carterae," PLoS ONE, vol. 3, no. 8, Article ID e2929, 2008.

[38] V. Ebenezer, W. A. Lim, and J.-S. Ki, "Effects of the algicides $\mathrm{CuSO}_{4}$ and $\mathrm{NaOCl}$ on various physiological parameters in the harmful dinoflagellate Cochlodinium polykrikoides," Journal of Applied Phycology, vol. 26, no. 6, pp. 2357-2365, 2014.

[39] L. M. Gaetke and C. K. Chow, "Copper toxicity, oxidative stress, and antioxidant nutrients," Toxicology, vol. 189, no. 1-2, pp. 147163, 2003.

[40] H. Qian, S. Yu, Z. Sun, X. Xie, W. Liu, and Z. Fu, "Effects of copper sulfate, hydrogen peroxide and N-phenyl-2-naphthylamine on oxidative stress and the expression of genes involved photosynthesis and microcystin disposition in Microcystis aeruginosa," Aquatic Toxicology, vol. 99, no. 3, pp. 405-412, 2010.

[41] A. D. Russell, "Similarities and differences in the responses of microorganisms to biocides," Journal of Antimicrobial Chemotherapy, vol. 52, no. 5, pp. 750-763, 2003.

[42] G. Bajszár and A. Dekonenko, "Stress-induced Hsp70 gene expression and inactivation of Cryptosporidium parvum oocysts by chlorine-based oxidants," Applied and Environmental Microbiology, vol. 76, no. 6, pp. 1732-1739, 2010.

[43] G. Coteur, B. Danis, S. W. Fowler, J.-L. Teyssié, P. Dubois, and M. Warnau, "Effects of PCBs on reactive oxygen species (ROS) production by the immune cells of Paracentrotus lividus (Echinodermata)," Marine Pollution Bulletin, vol. 42, no. 8, pp. 667-672, 2001.

[44] V. Ebenezer and J. S. Ki, "Evaluation of the sub-lethal toxicity of $\mathrm{Cu}, \mathrm{Pb}$, bisphenol $\mathrm{A}$ and polychlorinated biphenyl to the marine dinoflagellate Cochlodinium polykrikoides," Algae, vol. 27, pp. 63-70, 2012.

[45] S. H. Safe, "Polychlorinated biphenyls (PCBs): environmental impact, biochemical and toxic responses, and implications for risk assessment," Critical Reviews in Toxicology, vol. 24, no. 2, pp. 87-149, 1994.

[46] M. van den Berg, L. S. Birnbaum, M. Denison et al., "The 2005 World Health Organization reevaluation of human and mammalian toxic equivalency factors for dioxins and dioxinlike compounds," Toxicological Sciences, vol. 93, no. 2, pp. 223241, 2006.

[47] L. A. Carver, V. Jackiw, and C. A. Bradfield, "The 90-kDa heat shock protein is essential for Ah receptor signaling in a yeast expression system," The Journal of Biological Chemistry, vol. 269, no. 48, pp. 30109-30112, 1994.

[48] P. Coumailleau, L. Poellinger, J.-A. Gustafsson, and M. L. Whitelaw, "Definition of a minimal domain of the dioxin 
receptor that is associated with Hsp90 and maintains wild type ligand binding affinity and specificity," The Journal of Biological Chemistry, vol. 270, no. 42, pp. 25291-25300, 1995.

[49] J. McGuire, M. L. Whitelaw, I. Pongratz, J.-A. Gustafsson, and L. Poellinger, "A cellular factor stimulates ligand-dependent release of hsp90 from the basic helix-loop-helix dioxin receptor," Molecular and Cellular Biology, vol. 14, no. 4, pp. 24382446, 1994.

[50] I. Pongratz, G. G. F. Mason, and L. Poellinger, "Dual roles of the $90-\mathrm{kDa}$ heat shock protein hsp90 in modulating functional activities of the dioxin receptor: evidence that the dioxin receptor functionally belongs to a subclass of nuclear receptors which require hsp90 both for ligand binding activity and repression of intrinsic DNA binding activity," Journal of Biological Chemistry, vol. 267, no. 19, pp. 13728-13734, 1992.

[51] T. Liu, L. Pan, Y. Cai, and J. Miao, "Molecular cloning and sequence analysis of heat shock proteins 70 (HSP70) and 90 (HSP90) and their expression analysis when exposed to benzo(a)pyrene in the clam Ruditapes philippinarum," Gene, vol. 555, no. 2, pp. 108-118, 2015.

[52] W. B. Pratt and D. O. Toft, "Regulation of signaling protein function and trafficking by the hsp90/hsp70-based chaperone machinery," Experimental Biology and Medicine, vol. 228, no. 2, pp. 111-133, 2003. 

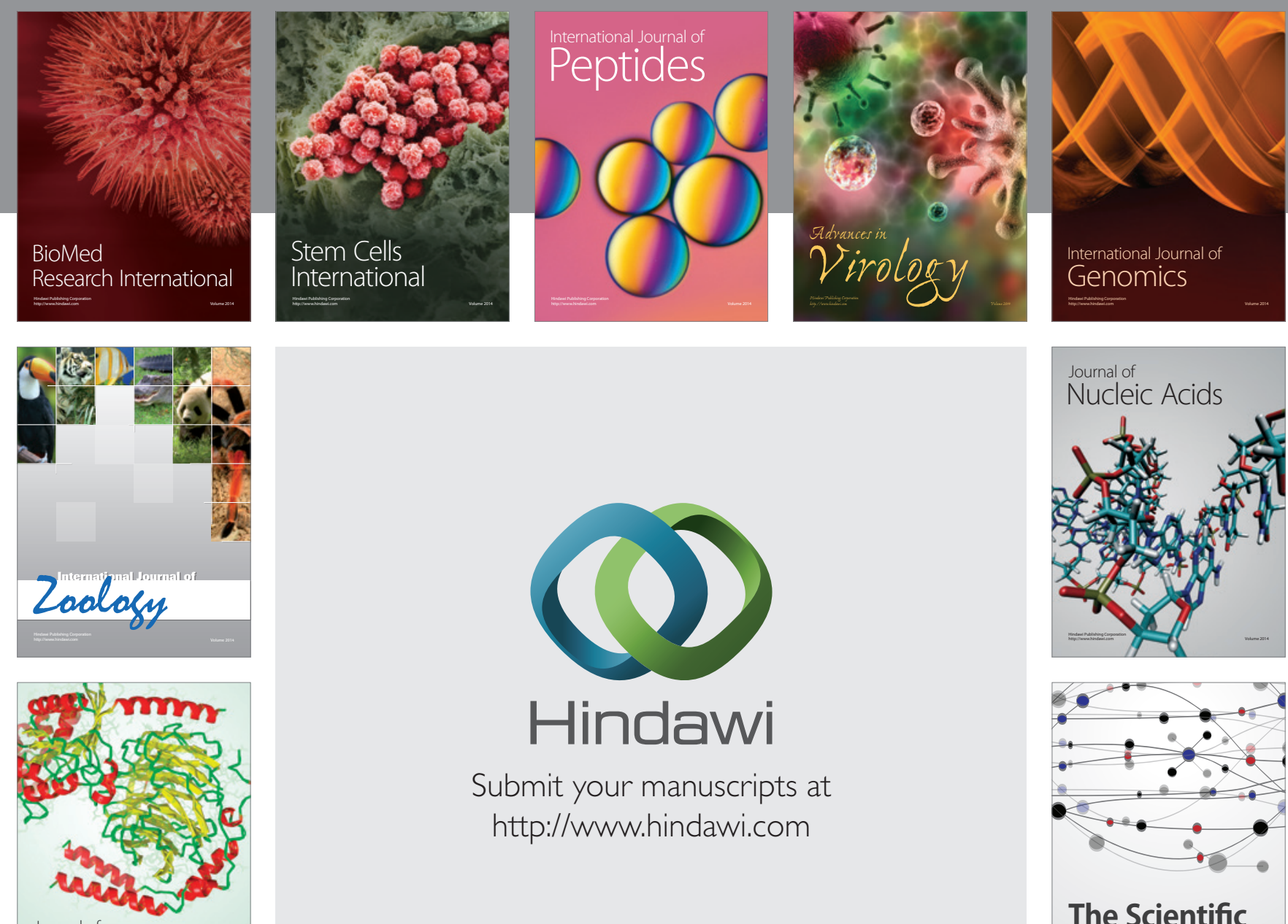

Submit your manuscripts at

http://www.hindawi.com

Journal of
Signal Transduction
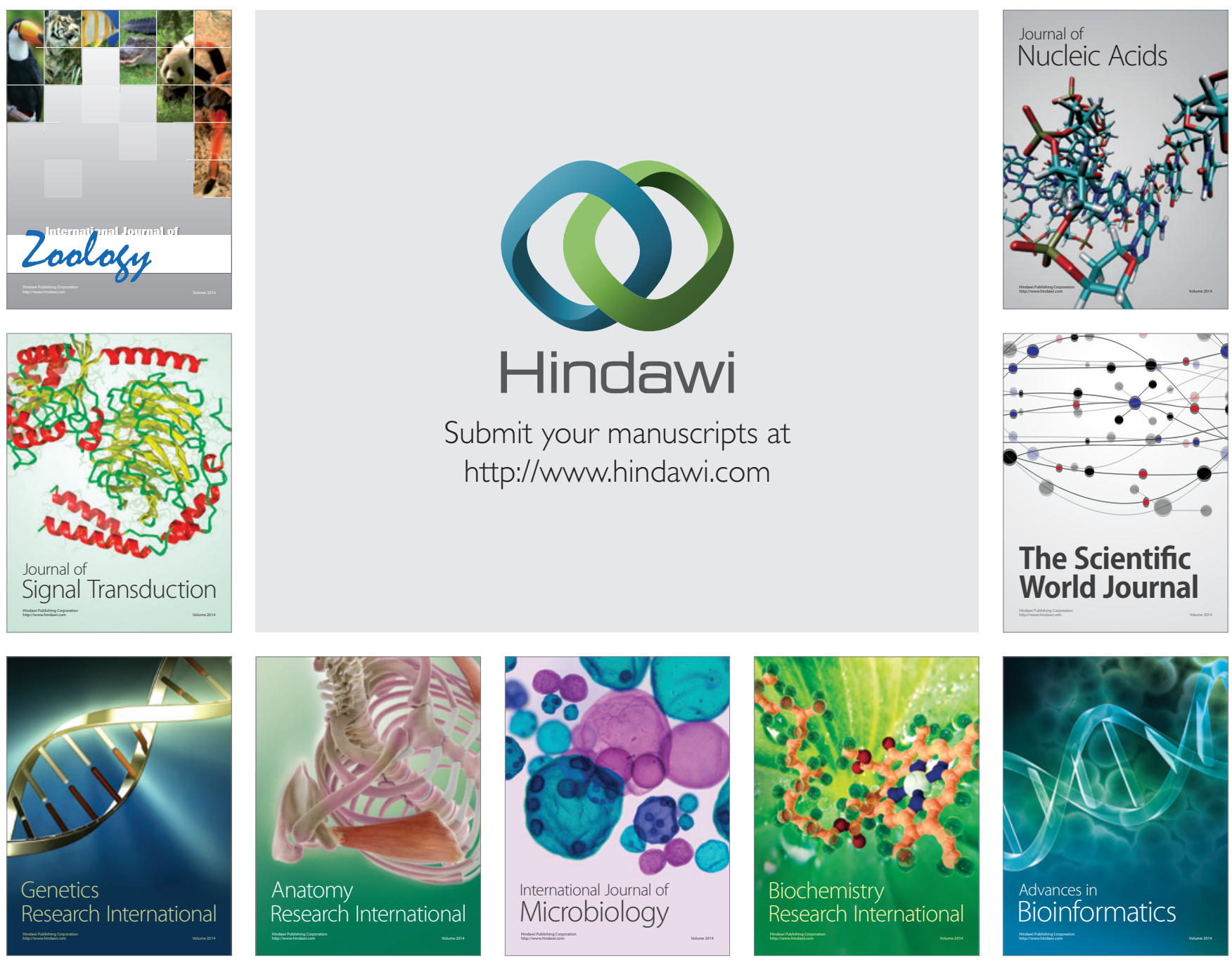

The Scientific World Journal
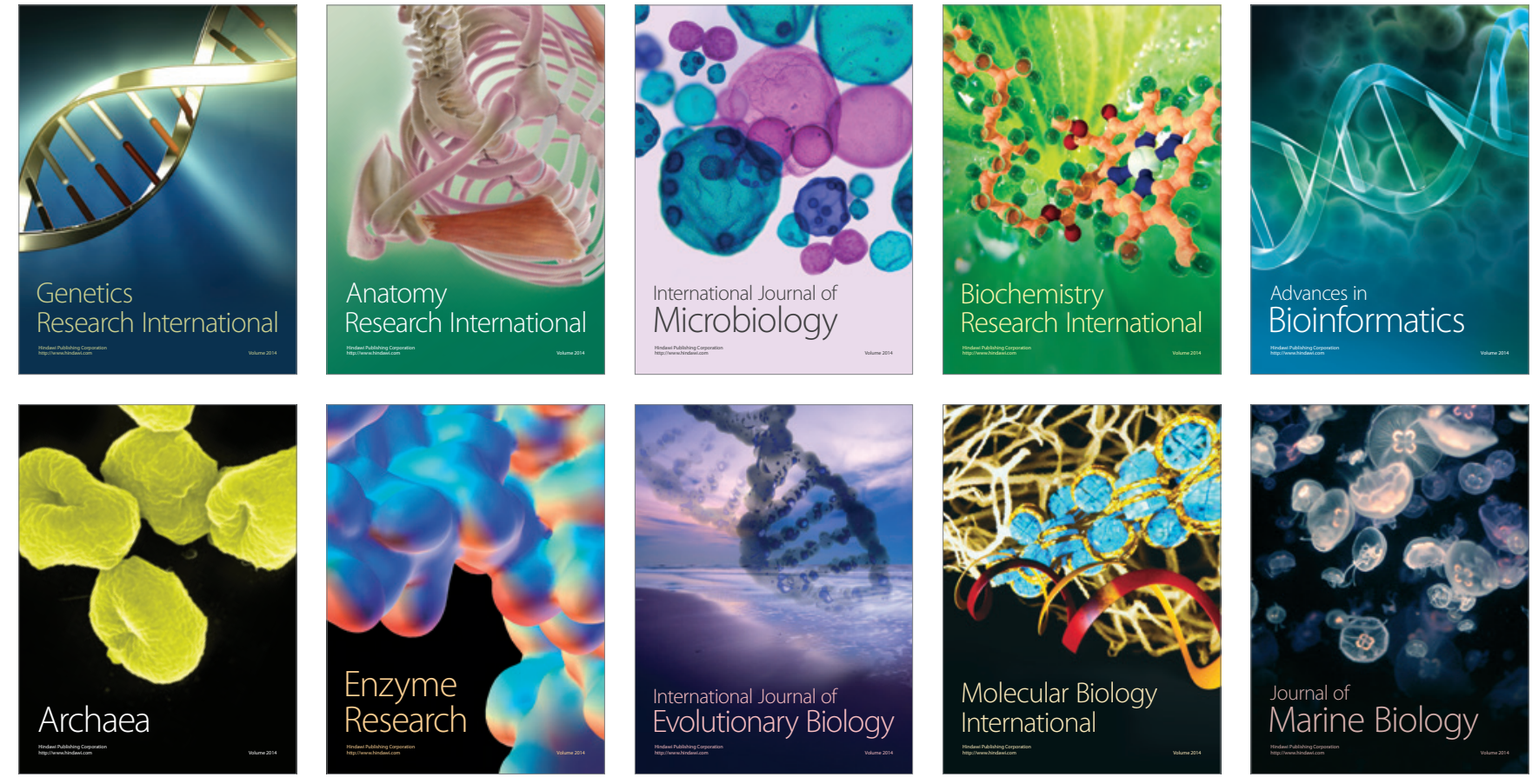